\title{
inventio
}

La génesis de la cultura universitaria en Morelos

Año 15, núm. 37, noviembre 2019

ISSN: 2007-1760 (impreso) 2448-9026 (digital) | DOI: 10.30973/inventio/2019.15.37/1

PENSAMIENTO UNIVERSITARIO

\section{El derecho al sol: acceso y disponibilidad de la luz natural en Querétaro}

\section{Luz Angélica Mondragón del Ángel}

ORCID: 0000-0001-5997-182X/luzmondragon215@gmail.com

Maestría en Arquitectura, Facultad de Ingeniería, Universidad Autónoma de Querétaro (UAQ)

\section{Avatar Flores Gutiérrez}

ORCID: 0000-0001-9581-3853/avatar.flores@uaq.mx

Profesor-investigador, Facultad de Ingeniería, Universidad Autónoma de Querétaro (UAQ)

\section{RESUMEN}

Este artículo presenta un análisis del acceso a la luz natural, desde tres enfoques: en la vida del ser humano, en las ciudades y como un derecho desde la perspectiva normativa, tomando como estudio de caso la ciudad de Querétaro. En él se busca recalcar la problemática actual de expansión de las ciudades y agotamiento de recursos, que se deriva en afectaciones a los requerimientos mínimos de diseño urbano, que se pensarían obvios, pero que hoy en día requieren del establecimiento de derechos para poder gozar de fuentes naturales, como el sol. Se exponen las políticas públicas que defienden este derecho en el ámbito nacional, además de las condiciones dadas por las legislaciones locales, en este caso en el estado de Querétaro, para construir planificando la incidencia solar y lumínica. Se proporciona la comprobación de la alta disponibilidad de luz natural en Querétaro y el surgimiento de nuevas temáticas, como la difusión de los derechos solares hacia la ciudadanía y las medidas a tomar para su aplicación.

PALABRAS CLAVE

ciudad; normativas; análisis lumínico; sustentabilidad; habitabilidad 
El objetivo principal del presente trabajo consiste en analizar los factores para el acceso a la luz natural. Se tiene en cuenta la política pública existente, partiendo del ámbito nacional hasta llegar a lo más concreto, en la ciudad de Querétaro. Asimismo, se busca evaluar la disponibilidad de iluminación natural en el sitio.

El interés por analizar estas variables consiste en dar a conocer la importancia del control en el diseño de espacios habitables a nivel masivo, es decir, en las ciudades, para que los habitantes puedan disponer del cumplimiento de sus derechos, entre ellos, los solares, que han surgido de la falta de congruencia actual entre la edificación y el medio ambiente. Como señala Knowles (1999), los edificios son indiferentes a la orientación solar, permanecen estáticos y no responden a los ritmos de su entorno, contrario a lo que sucede en la naturaleza, que parece contar con cuantiosos ejemplos basados en la exposición al sol.

El derecho al sol o el derecho al viento, más allá de estar fundamentados en el aprovechamiento de energías renovables que son producto del desarrollo sustentable, constituyen decretos para el ser humano en el nivel existencial. Ya sea por ahorro energético o por calidad de vida, el acceso a la luz solar es un área de la política pública que permite regular cómo y cuándo los vecinos pueden dar sombra a otros. Con el deterioro del ambiente urbano, el énfasis en el acceso a la luz solar se inclina hacia la calidad de vida (Knowles, 1999).

De acuerdo con los objetivos descritos, el artículo se estructura en cuatro secciones: la primera corresponde a la influencia de la luz natural en el ser humano y en el contexto de las ciudades actuales, estudio de caso en Querétaro; en la segunda se abordan los ordenamientos jurídicos que defienden el uso, aprovechamiento y acceso de la luz natural; en la tercera se efectúa un análisis de la disponibilidad de luz natural en el sitio, y por último, la sección final está dedicada a las conclusiones generales.

\section{Metodología}

El método utilizado se basa en la búsqueda bibliográfica de antecedentes en distintos medios, como libros, revistas, normas oficiales, internet, de los cuales se obtuvo la información necesaria para su estudio y presentación por secciones para facilitar la comprensión de cada enfoque.

Como método para examinar la disponibilidad de la luz natural en el sitio se obtuvo el análisis de los rangos de incidencia solar y lumínica en Querétaro mediante los programas Meteonorm y Climate Consultant.

\section{Luz natural, ser humano y ciudad}

La luz procedente del sol se puede definir como una fracción del espectro electromagnético de la radiación solar. Esta fracción es perceptible para el ojo humano, además de que le permite la visión, y de ella dependen los ciclos naturales de la materia viva en la Tierra (Guadarrama 
\& Bronfman Rubli, 2014). Sin duda, la luz natural y su aplicación tienen múltiples ventajas en la vida del ser humano: desde influir en el estado de ánimo de las personas hasta la recuperación de las debilidades físicas de un individuo, en referencia a ciertas enfermedades.

Las principales causas de la melancolía y el trastorno afectivo estacional son la falta de neuronas, luz solar y disminución de la serotonina. La exposición al sol puede estimular la producción de serotonina y reducir la posibilidad de tener melancolía; por lo tanto, la luz solar es crucial para permanecer saludable (Huang, Ruan \& Whang, 2016). "El ser humano segrega, gracias al ciclo día-noche, hormonas que mantienen en equilibrio al organismo. Si este ciclo se altera, alargando el periodo luminoso natural mediante el uso excesivo de la luz eléctrica por las noches o disminuyendo el flujo luminoso natural requerido por el día [...], provoca desajustes en la salud y en el bienestar psicológico de las personas" (Guadarrama \& Bronfman, 2014).

La luz natural viene acompañada de múltiples propiedades cuya influencia en el ser humano se debe controlar, como la radiación solar, el deslumbramiento, su dosificación y su impacto en la materia construida. Ésta ha sido creada por el ser humano como producto de su civilidad y necesidad; sin embargo, es innegable su preferencia por la iluminación natural sobre la artificial, pues aunque existen luminarias de espectro completo y de iluminación biodinámica que simulan la luz del sol, no hay una que iguale las características y beneficios completos que aporta la luz natural, como explica Sosa: "las diferencias visuales entre la luz natural y la luz artificial va más allá de las especificaciones técnicas de luz" (Sosa, 2016). Esto se debe a que la luz del sol incidente en el espacio habitado brinda una estrecha relación con las emociones y percepciones.

Por otro lado, en el mundo moderno la ciudad está obligada a responder ante la expectativa de una mejor calidad de vida de sus residentes ya que, debido al crecimiento demográfico y al fenómeno de la migración interna (rural-urbana y urbana-urbana), se enfrenta al reto de proveer mayor demanda de agua y suelo, desarrollo urbano, acceso a vivienda y otros servicios públicos (Varela, Ocegueda \& Castillo, 2017), además de proveer de los derechos y obligaciones que a cada ciudadano le corresponden.

Situándonos en Querétaro, la posición geográfica de la ciudad ha originado una intensa instalación de industrias y el acondicionamiento de áreas destinadas a servicios y vivien$\mathrm{da}$, lo que se ha traducido en el surgimiento de grandes conjuntos habitacionales y edificios habitacionales, tiendas departamentales y oficinas que han cambiado la estructura original compacta de la urbe. Este proceso se produjo entre 1960 y 1980, y con la ampliación del sector productivo continúa vigente y ha dado pie a la expansión de la mancha urbana (González Gómez, 2012).

La aglomeración urbana en Querétaro sigue extendiéndose y tiene como consecuencia una ocupación del suelo poco ordenada en creciente segregación (Göbel, 2015). Ante esta 
situación ¿qué pasa con los requerimientos básicos del habitar? Para arquitectos y diseñadores, claro está que su labor es proveer de estos requerimientos al igual que muchos otros factores que tienen que ver con el confort; para ellos la nueva tendencia de diseño avanzado sitúa al hombre como un participante del espacio y no como un usuario, por lo que no habría preocupación por pensar en cumplir con lo básico.

Considerando que en esta ciudad predominan tres tipologías constructivas, que son "las zonas residenciales; las monótonas construcciones de vivienda de interés social ordenadas linealmente, y las construcciones espontáneas y de autoconstrucción" (Göbel, 2015), nos preguntamos hasta qué punto se cuenta con las medidas de confort-habitabilidad necesarias y, específicamente, hasta qué punto se cuenta con los requerimientos indispensables de iluminación natural.

\section{Derechos solares}

En México, la política nacional de vivienda está a cargo de la Comisión Nacional de Vivienda (CONAVI), cuyo objetivo es formular y promover "que las autoridades competentes expidan, apliquen y mantengan en vigor y permanentemente actualizadas, disposiciones legales, normas oficiales mexicanas, códigos de procesos de edificación y/o reglamentos de construcción" (Comisión Nacional de Vivienda, 2010, p. I). Para ello creó el Código de Edificación de Vivienda (CEV), que promueve los lineamientos generales de la edificación de vivienda en todo el país, teniendo en consideración los reglamentos de construcción elaborados por los gobiernos locales.

El CEV contiene las consideraciones generales de sustentabilidad aplicables a la vivienda, dentro de las cuales se menciona el derecho al sol. En primer orden establece la necesidad de garantizar que en los terrenos colindantes al oriente, sur y poniente de una vivienda no se proyecten a futuro construcciones que puedan obstruir la incidencia solar e impidan su aprovechamiento mediante el diseño bioclimático (Comisión Nacional de Vivienda, 2010). Estos convenios se realizan entre los propietarios de lotes vecinos y se integran al título de propiedad, por lo que su incumplimiento puede implicar el pago de una indemnización al afectado. Para su cumplimiento, "se constituyen comités municipales para regular el uso de suelo y sancionar los permisos creando derechos solares" (Comisión Nacional de Vivienda, 2010, p. 317). Estos comités tienen la capacidad de limitar el desarrollo de construcciones u otro tipo de obstáculo que restrinja el acceso al sol de otros terrenos.

En el Reglamento General de Construcciones del Estado de Querétaro (1988), artículo 25, correspondiente a la sección de acondicionamiento y confort ambiental, se menciona que los locales en las edificaciones deben contar con los medios que aseguren la iluminación diurna y nocturna para sus ocupantes, además de que los espacios habitables, cocinas domésticas y cuartos de hospitales deben estar provistos de iluminación natural por medio de ventanas que den directamente al exterior.

Año 15, núm. 37, noviembre 2019 
Asimismo, se establece el porcentaje mínimo para las ventanas de acuerdo con el área del local y según su orientación: norte $10 \%$, sur 12\%, este 10\% y $8 \%$ para el poniente. Se señala que, para fachadas de colindancia, la iluminación podrá ser mediante bloques de vidrio, sin que esto altere los requerimientos mínimos establecidos para tamaño de ventanas y domoso tragaluces (Reglamento General de Construcciones del Estado de Querétaro, 1988). Además, se establecen los niveles de iluminación para las construcciones de tipo habitacional: 75 lux para locales habitables y de servicio y 50 lux para circulaciones horizontales y verticales (Reglamento General de Construcciones del Estado de Querétaro , 1988, art. 26).

El Código Urbano del Estado de Querétaro (2012, art. 349) menciona algunas medidas a tratar para evitar las obstrucciones lumínicas en las edificaciones, como que "los propietarios de las construcciones de dos o más niveles que ejecuten fachadas de colindancia que formen parte de los paramentos de patio de iluminación y ventilación de construcciones vecinas, deberán tener acabados impermeables y de color claro [sic]".

Ahora bien, las medidas y políticas en cuanto al derecho y preocupación para que los inmuebles gocen de los beneficios del sol existen y se encuentran referenciados en los documentos correspondientes al ámbito constructivo y legal. Por lo tanto, si se siguieran las normativas expuestas no tendrían razón de ser los espacios escasos de luz natural, a menos que fuera imposible proveerlos por condiciones climáticas, topográficas o de obstrucciones.

A continuación se analiza la disponibilidad lumínica en Querétaro, con la finalidad de comprobar que esta zona cuenta con un alto de nivel de radiación solar para su aprovechamiento y establecer estrategias de diseño adecuadas, con base en la normatividad, para que cualquier espacio sea iluminado de manera natural.

\section{Disponibilidad de luz natural en el sitio}

La disponibilidad y características de la luz natural dependen de la latitud, meteorología, época del año y momento del día (Instituto para la Diversificación y Ahorro de Energía, 2005); por lo tanto, la cantidad de luz natural recibida varía con la situación del clima y la calidad del aire, que también afectan la intensidad y duración de la luz natural.

De esta manera, las estrategias de iluminación natural y aprovechamiento de la luz solar dependen de su disponibilidad, de las condiciones de iluminación que existen en los espacios, de las obstrucciones que bloquean el paso de la luz, de las edificaciones colindantes y de la topografía del terreno, que puede modificar la cantidad de luz a ciertas horas del día.

La ubicación de la ciudad de Querétaro le permite una gran disponibilidad de radiación solar, a diferencia de otras ciudades ubicadas al norte o sur del continente. Y no sólo Querétaro posee esta ventaja, ya que nuestro país se ve ampliamente favorecido con una alta incidencia de radiación solar en gran parte del territorio, debido a su cercanía con el Ecuador. En promedio tiene valores de 5 a $6 \mathrm{kWh} / \mathrm{m}^{2} /$ día durante el verano (Tejeda \& Gómez, 2015).

Año 15, núm. 37, noviembre 2019 
Para el análisis de la disponibilidad de la luz natural de acuerdo con el lugar, se realizó un estudio de las características de luz natural para el municipio de Querétaro.

Figura 1

Niveles de radiación mensual $\left(\mathrm{kWh} / \mathrm{m}^{2}\right)$ para Querétaro

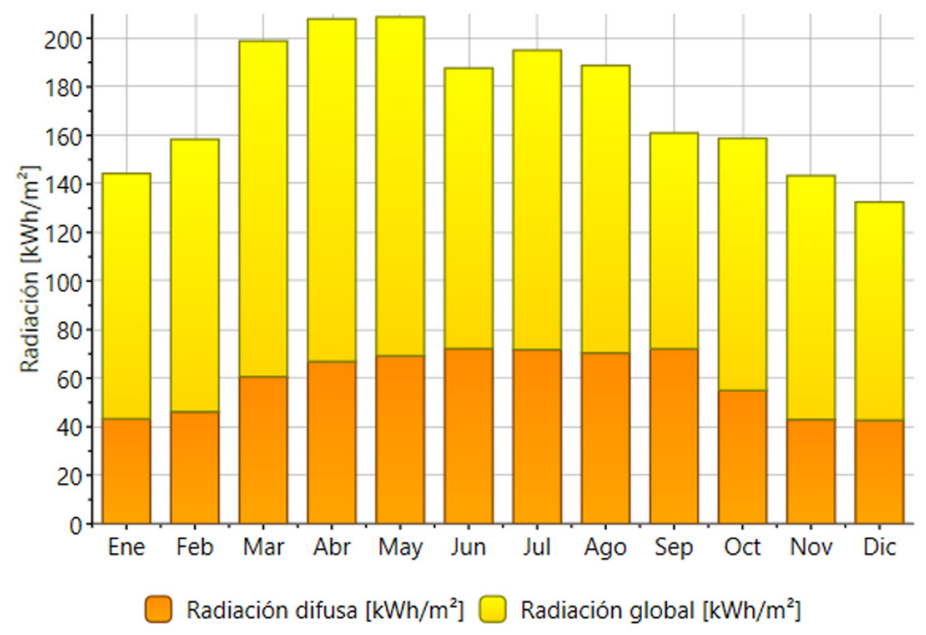

Fuente: Elaboración propia mediante Meteonorm, 2018

Figura 2

Radiación global diaria $\left(\mathrm{kWh} / \mathrm{m}^{2}\right)$ para Querétaro

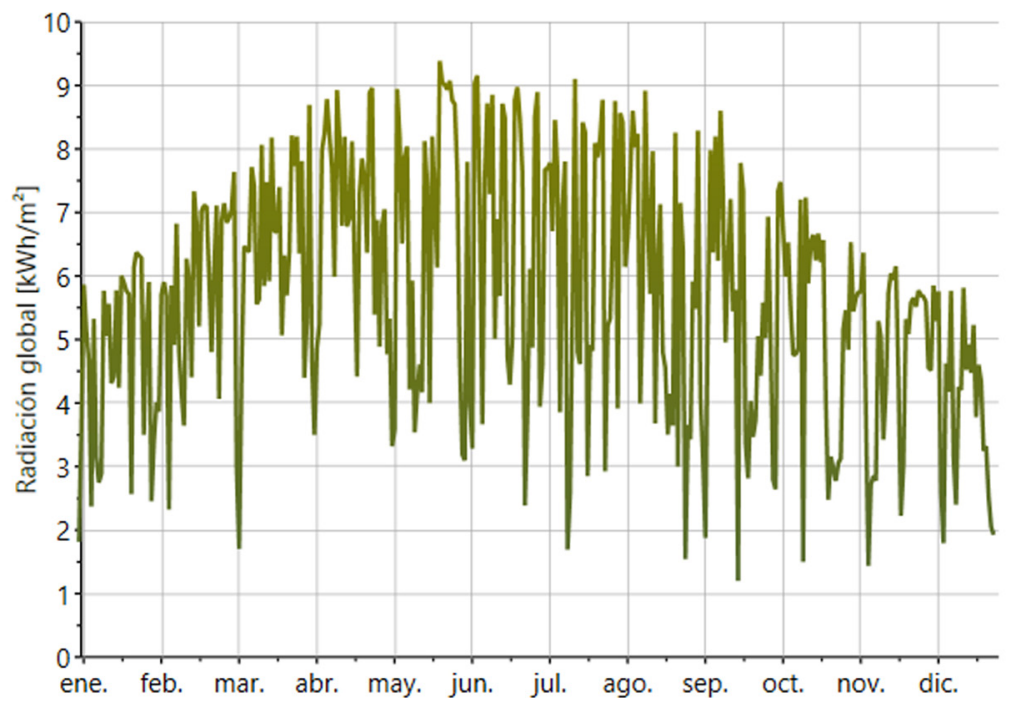

Fuente: Elaboración propia mediante Meteonorm, 2018

Año 15, núm. 37, noviembre 2019 
En la gráfica de radiación mensual (figura 1) se observa que la máxima radiación global se recibe en los meses de marzo, abril y mayo, con valores por encima de los $200 \mathrm{~kW} / \mathrm{m}^{2}$. De igual manera, en la gráfica de radiación global diaria (figura 2) se aprecia que en los mismos meses se tienen días que llegan a los $9 \mathrm{~kW} / \mathrm{m}^{2}$, lo cual refleja la gran cantidad de radiación solar que se recibe en el municipio. Por estas condiciones, es necesario protegerse de la radiación excesiva, pero también se debe considerar aprovecharla y mediarla en las edificaciones con mayor nivel de permanencia, como es el caso de la vivienda.

En la figura 3 se muestran los valores mensuales de las horas recibidas de radiación solar, y con ello, la duración de la luz del día en el municipio de Querétaro. Se obtiene un promedio anual de 12 horas por día de duración astronómica de la radiación solar, la cual nos permite realizar nuestras actividades cotidianas.

Figura 3

\section{Promedio por mes de la duración de la radiación solar (h) en Querétaro}

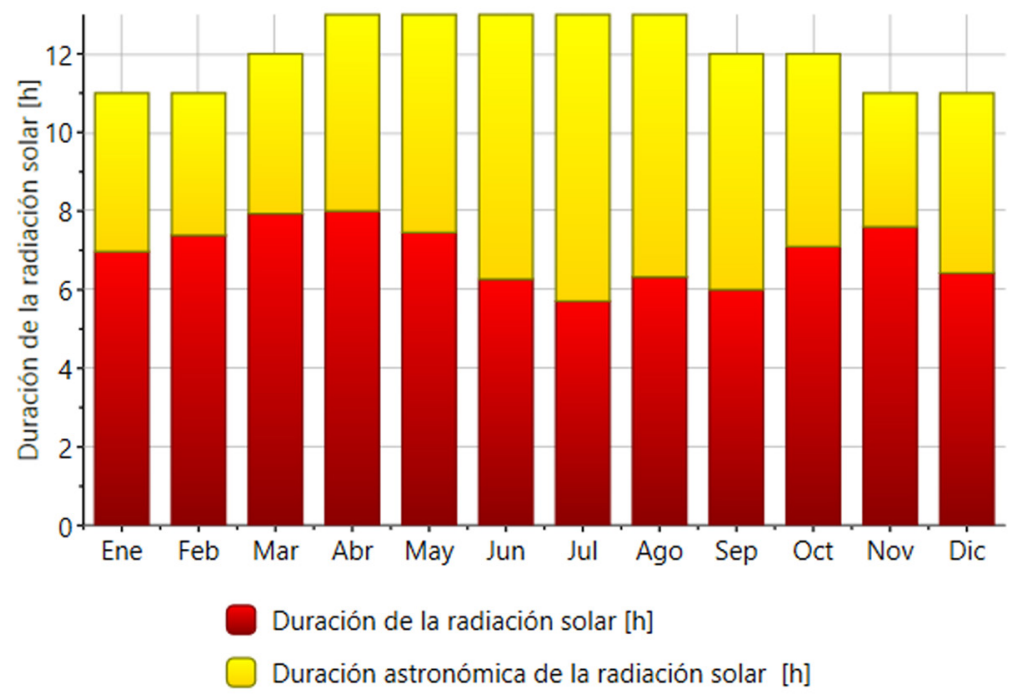

Fuente: Elaboración propia mediante Meteonorm, 2018

Otro factor por considerar en el análisis de la incidencia de la radiación solar, y con ello de la disponibilidad de la luz natural en el municipio de Querétaro, es el porcentaje de días nublados y soleados (figura 4), donde se observa una disponibilidad lumínica de $82 \%$ a lo largo del año.

Año 15, núm. 37, noviembre 2019

ISSN: 2007-1760 (impreso) 2448-9026 (digital) | DoI: 10.30973/inventio/2019.15.37/1 
Figura 4

Porcentaje de días nublados y soleados en el año en Querétaro

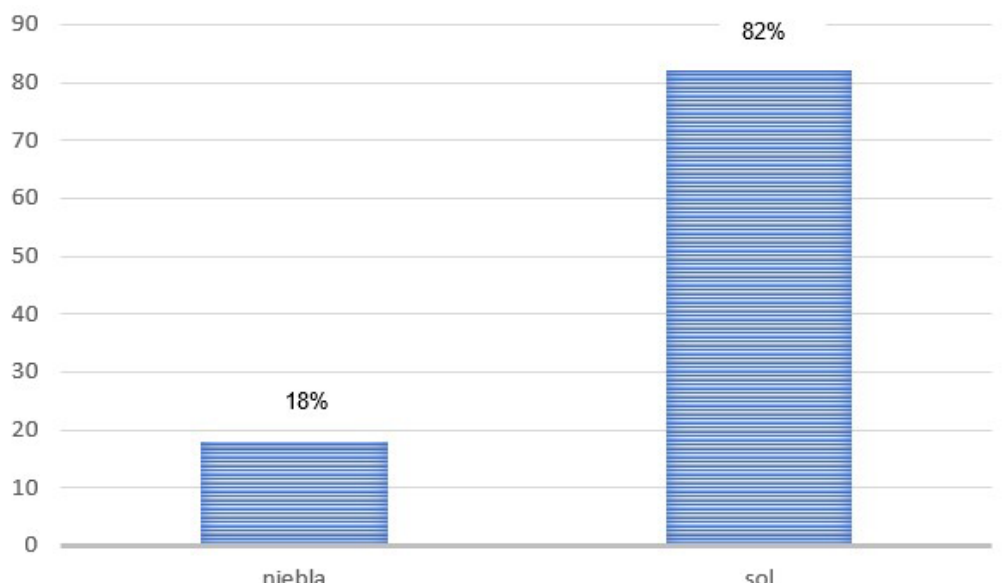

Porcentaje de días nublados y soleados en el año en Querétaro, $20^{\circ} 30^{\prime}$ y $20^{\circ} 56^{\prime}$ de latitud norte; $100^{\circ} 17^{\prime}$ y $100^{\circ} 36^{\prime}$ de longitud oeste, con una altitud entre 1700 y $2800 \mathrm{~m}$

Fuente: Elaboración propia con datos de weatherbase.com, 2018

Tabla 1

Niveles de luz comunes en exterior de fuentes naturales

\begin{tabular}{|l|r|}
\hline Condición & lluminancia (lux) \\
\hline Luz de sol & 107,527 \\
\hline Plena luz del día & 10,752 \\
\hline Día nublado & 1,075 \\
\hline Día oscuro & 107 \\
\hline Crepúsculo & 10.8 \\
\hline Crepúsculo profundo & 1.08 \\
\hline Luna llena & 0.108 \\
\hline Luna creciente & 0.0108 \\
\hline Luz de las estrellas & 0.0011 \\
\hline Noche nublada & 0.0001 \\
\hline
\end{tabular}

Fuente: Elaboración propia, con datos de la Association of Universities for Research in Astronomy (2016).

Año 15, núm. 37, noviembre 2019 
Para tener una referencia de la cantidad de iluminación en las horas diurnas del lugar, se tienen los niveles de luz de fuentes naturales medidos en luxes (tabla 1), que permiten comparar y comprender los rangos mensuales de iluminación en el sitio (figura 5). Se observa que el mayor promedio por mes (barra amarilla) es de 83,000 lux en marzo, y el menor, de 65,000 lux en junio.

Figura 5

Rangos mensuales de iluminación para Querétaro según las horas iluminadas (lux)

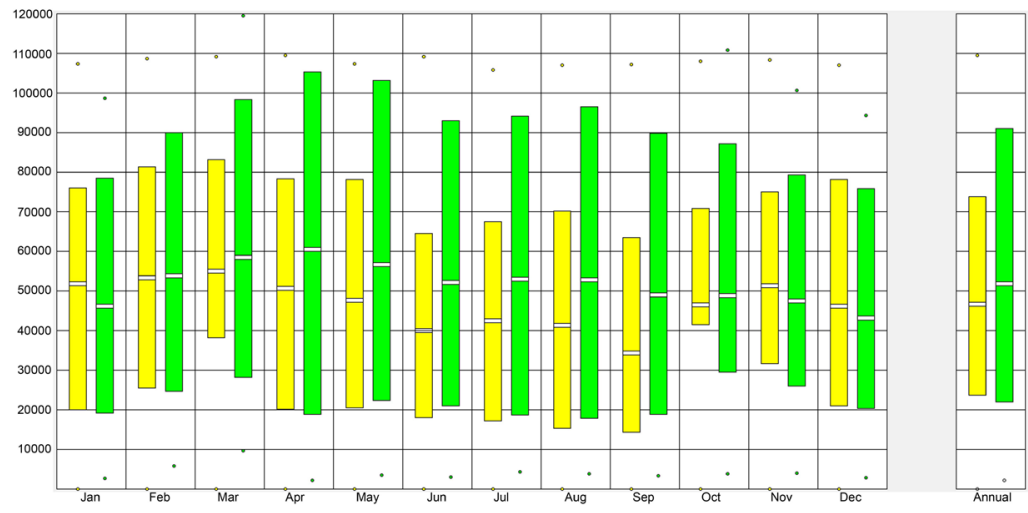

Fuente: Elaboración propia mediante Climate Consultant 6.0, 2018

A lo largo del presente estudio se observa que en el sitio se tienen óptimas condiciones lumínicas y se cuenta con la legislación pertinente para que cualquier habitante de un espacio ejerza su derecho al sol; sin embargo, la creciente demanda de servicios -entre ellos, la vivienda, el crecimiento demográfico y la atracción continua que generan las ciudades, en este caso Querétaro- pueden desencadenar faltas graves de diseño arquitectónico que afectan directamente el bienestar de los habitantes, como anteriormente se señalaba. En tal situación es donde radica la importancia de difundir y conocer las normativas, el sitio y las características de las ciudades en crecimiento.

\section{Conclusiones}

Los principales factores analizados en el estudio sobre el acceso a la luz natural fueron la importancia de su influencia en el ser humano, que le causa el bienestar con su adecuada dosificación y control; el papel de la luz natural en la planificación de espacios específicos y en la escala urbana; por último, su disponibilidad en el sitio.

Respecto a los cuestionamientos en un inicio planteados, se puede concluir que en un estado ideal y conforme a la literatura citada (decretos sobre los derechos solares en México), esta fuente de energía es para todos por derecho. Sin embargo, si bien existen ordenamien- 
tos que defienden estos derechos cuyo incumplimiento tiene una sanción, basta con caminar por las ciudades y observar que existen edificios, infraestructura y elementos urbanos que obstruyen el aprovechamiento de la luz natural en locales habitables. Incluso si no hubiera tales obstrucciones sería posible encontrar casos en los que la falta de un diseño adecuado en la vivienda ocasiona que se tengan locales privados de iluminación natural dentro de ella. Por lo tanto, este trabajo brinda la oportunidad de desarrollar investigaciones para comprobar lo que empíricamente se ha mencionado líneas atrás, ya que las normativas existen pero la ciudadanía desconoce estos decretos o, en un escenario más crítico, no los aplica. Por ello es necesaria la tarea de divulgación. Ante la expansión de las ciudades, el crecimiento demográfico y el agotamiento de los recursos y las fuentes de energía no renovable, es tiempo de considerar conscientemente al sol.

Debido a su emplazamiento geográfico, algunos lugares gozan de mayor o menor incidencia de energía solar. Nuestro país se ve favorecido en este aspecto. Los datos obtenidos en el análisis de la disponibilidad de la luz natural en la ciudad de Querétaro son de gran interés para compararlos con otros sitios, llegar a conclusiones más detalladas sobre la cantidad de energía y los beneficios que se pueden obtener con la luz natural y cómo emplearla en el diseño de espacios. En este sentido, el aprovechar fuentes de energía renovable es una urgencia actual, pero también es significativo saber que somos privilegiados por la cantidad de luz natural que tenemos en la zona de estudio y que debemos aplicar los ordenamientos legales para que sea una obligación el uso y aprovechamiento de la radiación solar-lumínica en México.

Para que los derechos solares existan haciendo énfasis en la iluminación natural se requiere de: 1) normativas aplicables con carácter obligatorio y 2) accesibilidad solar, la cual depende de la planificación arquitectónica (espacial) en relación con la disponibilidad lumínica de la zona y el análisis lumínico para cada edificio en particular, con respecto a la orientación, las posibles obstrucciones y la topografía específica del emplazamiento.

La planeación urbana, por lo tanto, necesita que las ciudades sean diseñadas pensando más allá de los usos de suelo por metro cuadrado, es decir, considerando las alturas y las formas volumétricas para establecer adecuadas zonificaciones en los espacios habitables que garanticen el acceso a la radiación solar y no comprometan la calidad de vida de sus ocupantes.

\section{Referencias}

Association of Universities for Research in Astronomy, National Optical Astronomy Observatory (2016). Niveles deiluminación recomendados. https://www.noao.edu/education/QLTkit/ es/Safety_Activity_Poster/LightLevels_outdoor+indoor_es.pdf

Código Urbano del Estado de Querétaro (2012). Periódico Oficial del Gobierno del Estado de Querétaro "La Sombra de Arteaga", 31 de mayo de 2012 (PO No. 27). https://www. 
tribunalqro.gob.mx/biblio/leeDoc.php?cual=835\&tabla=tbiblioteca_historial

Comisión Nacional de Vivienda (2010). Código de edificación de vivienda. México: Gobierno Federal. https://www.gob.mx/conavi/documentos/codigo-de-edificacion-devivienda

Göbel, C. (2015). Una visión alemana de los modelos de ciudad: el caso de Querétaro. Gremium, 2, 47-60. https://editorialrestauro.com.mx/una-vision-alemana-de-los-modelos-deciudad-el-caso-de-queretaro/

González Gómez, C. I. (15 de julio, 2012). Querétaro metropolitano, una lectura sobre su nuevo crecimiento. Trabajo presentado en el International Congress of Americanists, Building Dialogues in the Americas, Viena.

Guadarrama, C. \& Bronfman Rubli, D. (2014). Sobre luz natural en arquitectura. Bitácora arquitectura, 29, 76-83.

Huang, J. T., Ruan, S. J. \& Whang, J. W. (2016). Intelligent natural light illumination system. Conference on Intelligent Green Building and Smart Grid (IGBSG), Praga, 1-6. https://doi. org/10.1109/IGBSG.2016.7539421

Instituto para la Diversificación y Ahorro de la Energía (2005). Guía Técnica: Aprovechamiento de la luz natural en la iluminación de edificios. Madrid, IDAE.

Knowles, R. L. (1999). The solar envelope. Los Ángeles: University of Southern California. http:// www-bcf.usc.edu/ rknowles/sol_env.html

Reglamento General de Construcciones del Estado de Querétaro (1988). Periódico Oficial del Gobierno del Estado de Querétaro "La Sombra de Arteaga". http://www.ordenjuridico. gob.mx/fichaOrdenamiento.php?idArchivo=31760\&ambito=ESTATAL

Sosa, L. (2016). La luz artificial como tecnología de simulación de la luz natural: evaluación de fiabilidad de los parámetros perceptivos y de confort (Tesis de Maestría). Universitat Politécnica de Catalunya, Barcelona. https://upcommons.upc.edu/handle/2117/100928

Tejeda Martínez, A. \& Gómez-Azpeitia, G. (comps.) (2015). Prontuario solar de México. Colima: Universidad de Colima, Universidad Veracruzana. http://ww.ucol.mx/content/publicacionesenlinea/adjuntos/Prontuario-solar-de-Mexico-(noviembre-de-2015)_6.pdf

Varela, R., Ocegueda, J. M. \& Castillo, R. (2017). Migración interna en México y causas de su movilidad. Perfiles Latinoamericanos, 49, 141-167. http://www.redalyc.org/articulo. oa?id=11549670007

Año 15, núm. 37, noviembre 2019 